\title{
RAPID ROOM TEMPERATURE LIQUID PHASE SYNTHESIS OF DIETHYL 2-((4-NITROANILINO) METHYLENE)MALONATE
}

\author{
HERNÁN VALLE ${ }^{a}$, RAMALINGA VISWANATHAN MANGALARAJA ${ }^{a,{ }^{*},}$, BERNABÉ L. RIVAS ${ }^{b}$, \\ JOSÉ BECERRA ${ }^{c}$, AND SELVARAJ NAVEENRAJ ${ }^{a}$ \\ a Advanced Ceramics and Nanotechnology Laboratory, Faculty of Engineering, University of Concepcion, Casilla 160, Chile. \\ ${ }^{b}$ Polymer Department, Faculty of Chemistry, University of Concepción, Casilla 160, Chile. \\ ${ }^{c}$ Laboratorio de Química de Productos Naturales, Facultad de Ciencias Naturales y Oceanográficas, Universidad de Concepción, Casilla 160, Chile.
}

\section{ABSTRACT}

Diethyl 2-((4-nitroanilino)methylene)malonate [4-NANM-E] is an important molecule owing to its role of precursor in the multistage synthesis of several quinoline derivatives possessing biological activities such as antiviral, immunosuppressive, anticancer and photoprotector. This molecule is usually synthesized by a nucleophilic vinyl substitution $\left(\mathrm{S}_{\mathrm{N}} \mathrm{V}\right)$ between 4-nitroaniline and diethylethoxymethylene malonate (EMA). Although several procedures are available to synthesize 4-NANM-E in liquid phase, more convenient method is necessary to synthesize in less reaction time and at room temperature. In this study, it is demonstrated that equimolar amounts of EMA and 4-nitroaniline dissolved in alcoholic $\mathrm{KOH}$ react within a few seconds at room temperature to produce 4-NANM-E which is purified by simple filtration after acidification with aqueous $\mathrm{HCl}$ and washing with alcohol. The reaction has the yield varying at the range $45-53 \%$ when it occurs in ethanol, 2-propanol, 2-butanol or 2-pentanol. Therefore, this synthesis method is an excellent alternative to produce 4-NANM-E on an industrial scale.

Keywords: Anilinomethylenemalonate, Room temperature synthesis, Quinoline, Methanolysis.

\section{INTRODUCTION}

Aryl substituted derivatives of diethyl anilinomethylenemalonate are among the most commonly used precursors in the multistage synthesis of approved and developing quinolinic drugs. From the anilinomethylenemalonate derivatives, the respective 4-quinolone intermediates are obtained by the Gould-Jacobs reaction or thermal cycling. To date, several researchers have used diethyl 2-(4-nitroanilino)methylenemalonate [4-NANM-E] as a precursor in the synthesis of different 6-nitro-4-quinolone derivatives possessing medicinal properties, such as antibacterial, ${ }^{1,2}$ antiviral, immunostimulant, immunosuppressant, photoprotector, cognitive enhancer and calcium-activated potassium channels blocker (study of sleep disorders). ${ }^{3-11}$ Anilinomethylenemalonate precursors are frequently obtained by nucleophilic addition of a substituted aniline to the polarized and electron deficient double bond of diethylethoxymethylene malonate (EMA), which forms a transitory carbanion that finally undergoes the elimination of the ethoxyl group (leaving group with charge negative). ${ }^{12}$ The rate of this reaction nucleophilic vinyl substitution $\left(\mathrm{S}_{\mathrm{N}} \mathrm{V}\right)$ is less as the electron-attracting force of the substituent on the aromatic ring increases and vice versa. In the particular case of the synthesis of 4-NANM-E, the electron-attracting effect of the nitro group on 4-nitroaniline, and the delocalization of nitrogen lone pair into the aromatic ring (and nitro substituent) cause a lower electron density on the nitrogen atom and also the lower pKa value when compared with other anilines, which makes it a weak nucleophile and base that requires more time and heating to react with EMA. ${ }^{13-16}$ As a consequence, the large-scale production of 4-NANM-E using conventional methodologies would imply high energy and time costs.

Although 4-NANM-E has been synthesized by the solvent-free synthesis using microwaves and $\mathrm{K}_{2} \mathrm{CO}_{3} / \mathrm{Al}_{2} \mathrm{O}_{3}$ as a catalyst, ${ }^{17}$ this type of synthesis requires very sophisticated and expensive reactors which also have some limitations in their large-scale synthesis of molecules. Furthermore, the use of microwave systems in organic synthesis is controversial since many researchers found that the reactors used in most cases did not allow precise control over power and temperature which is essential for good reproducibility of results. ${ }^{18}$ In order to contribute a more convenient alternative methodology for the preparation of 4-NANM-E, this study presents a fast and efficient method of liquid phase synthesis at room temperature of this useful synthetic intermediate, based on the use of alcohol- $\mathrm{KOH}$ as the reaction medium in the condensation between EMA and 4-nitroaniline. No studies on the synthesis of 4-NANM-E using alcohol-KOH as reaction medium at room temperature were found in the literature.

\section{EXPERIMENTAL}

\subsection{Materials and reagents}

Diethylethoxymethylene malonate (EMA, $\geq 98 \%$ ), 4-nitroaniline (4-NA, $\geq 98 \%$ ), potassium hydroxide $(\mathrm{KOH}$, pellets $>95 \%), \mathrm{HCl}$ aqueous solution (6M), solvents of analytical grade: methanol, ethanol, 2-propanol, 2-butanol and 2-pentanol were supplied by Merck. All reagents and solvents were used without further purification. The $\mathrm{pH}$ of the reaction mixture was measured with universal indicator paper ( $\mathrm{pH} 1-10$, Merck). Whatman $\mathbf{N}^{\circ} 2$ filter paper was used for vacuum filtration. The thin layer chromatography (TLC) technique was applied to confirm the identity and purity of the expected product. TLC was developed using aluminum chromateplates $(10 \times 5 \mathrm{~cm})$ with silica gel 60 F-254 (Merck), hexane-ethyl acetate (1: 1) as eluent, and a ultraviolet lamp (CAMAG, 254 and $366 \mathrm{~nm}$ ) was used as revelator for the chromatograms.

2.2. Synthesis of 4-NANM-E using alcohol-KOH without heating

$40 \mathrm{~mL}$ of 2-propanol and 2 pellets of $\mathrm{KOH}$ ( $300 \mathrm{mg}$, approximately) were mixed in a $100 \mathrm{~mL}$ open beaker (borosilicate glass) at room temperature $\left(25^{\circ} \mathrm{C}\right)$ using a magnetic stirrer. On continuous stirring, $691 \mathrm{mg}$ of 4-nitroaniline ( $5 \mathrm{mmol}$ ) was added to the 2-propanol-KOH solution. After the complete dissolution of 4-nitroaniline, $1 \mathrm{~mL}$ of EMA ( $5 \mathrm{mmol}$ ) was added. After $\sim 5$ minutes, the above reaction mixture was acidified with $6 \mathrm{M} \mathrm{HCl}$ until it reaches the $\mathrm{pH}$ value of 3 , and then the precipitate formed was filtered under vacuum, washed with 2-propanol, and finally dried in an oven with air circulation at $60^{\circ} \mathrm{C}$. For comparison purposes, the same procedure as above was repeated four times by changing the solvent of the $\mathrm{KOH}$, 2-propanol, for the following solvents: methanol, ethanol, 2-butanol, and 2-pentanol.

2.3. Synthesis of 4-NANM-E without solvent and using conventional heating

Standard 4-NANM-E was synthesized by following the Riegel's procedure. ${ }^{14}$

2.4. Characterization

${ }^{1} \mathrm{H}(400 \mathrm{MHz})$ and ${ }^{13} \mathrm{C}(100 \mathrm{MHz}) \mathrm{NMR}$ (nuclear magnetic resonance) spectra were obtained on a Bruker $400 \mathrm{NMR}$ spectrometer as solutions in deuterated DMSO (DMSO- $\mathrm{d}_{6}$ ) at a concentration of $30 \mathrm{mg} \mathrm{mL}^{-1}$ and using TMS as an internal standard. Infrared spectra (FTIR) were obtained with a Spectrum One spectrometer (PerkinElmer) using the $\mathrm{KBr}$ pellet technique.

\section{RESULTS AND DISCUSSION}

The dissolution of 4-nitroaniline and 2-propanol-KOH showed a "translucent brownish yellow" color, but within a few seconds after adding EMA (less than 10 seconds), it changed to "turbid reddish orange" followed by the formation of a light-colored precipitate. The same happens in cases where ethanol, 2-butanol, and 2-pentanol were used, although in the last two cases, a 
"gel-like" suspension was formed due to the absorption of the solvent from the reaction mixture. When methanol- $\mathrm{KOH}$ was used, the "translucent brownish yellow" color persisted even after the addition of EMA, and the formation of the precipitate was slower (after 10 minutes at rest) than the previous cases along with the lower yield. During the acidification step (Scheme 1), at $\mathrm{pH} 3$ all reaction mixtures were discolored and a "beige" precipitate was more clearly observed in the bottom of the beakers.<smiles>[NH3+]c1ccc([N+](=O)[O-])cc1</smiles>

4-Nitroaniline<smiles>CCOC=C(C(=O)OCC)C(=O)OCC</smiles>

EMA
1) $\mathrm{KOH} / 2-\mathrm{PrOH}, \sim 25^{\circ} \mathrm{C}$

2) Stirring $x \sim 10 \mathrm{sec}$

3) $\mathrm{HCl}_{(\text {aq) }}$, filtration<smiles>CCOC(=O)C(=CNc1ccc([N+](=O)[O-])cc1)C(=O)OCC</smiles>

4-NANME

Scheme 1. Synthesis of 4-NANM-E using 2-propanol-KOH without heating.

All the precipitates (post-acidification) were soluble in THF and DMSO, and were comparatively analyzed by TLC with the standard 4-NANM-E which was previously synthesized by Riegel's method and well characterized with NMR and IR spectroscopy. A single spot was observed for each precipitate with the same Rf value except for the precipitate formed with methanol-KOH system in which three spots were observed. The absence of spots with Rf value corresponding to the reagents confirmed the efficient purification of the product by washing with alcohol. Among the three spots observed in the methanol$\mathrm{KOH}$ system, the one with the highest fluorescence has the lower Rf value than that of the standard 4-NANM-E as well as the reactant EMA and slightly higher value than that of the other reactant 4-nitroaniline.

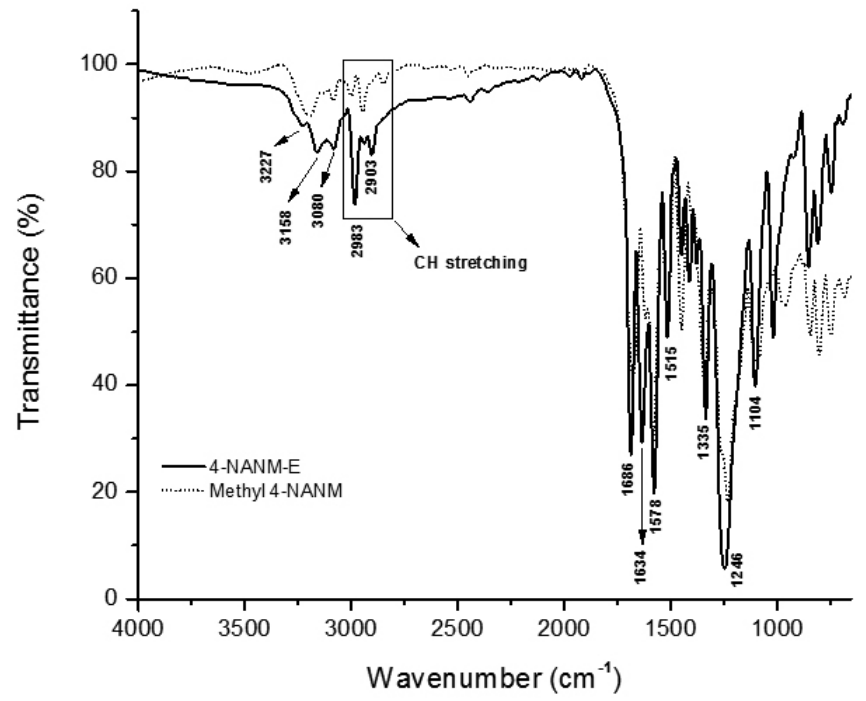

Figure 1. FTIR spectra of the compounds 4-NANM-E (solid line) and 4-methyl-NANM (dotted line). The stretching vibrations (v) C-H of 4-methylNANM (rectangular area) have bands of less intensity than the 4-NANM-E.

While comparing the IR spectra of the synthesized products with the standard 4-NANM-E, only the product obtained with methanol-KOH system differed mainly in the lower intensity of the $\mathrm{CH}$ stretching vibration between 2850-3000 $\mathrm{cm}^{-1}$ (Figure 1, Table 1) which suggested the presence of methyl ester groups instead of ethyl ester ones. It explains the higher polarity of the product obtained with methanol- $\mathrm{KOH}$ which showed a lower Rf value than that of the standard 4-NANM-E in TLC. Among all the signals shown in the IR spectra of Figure 1 and Table 1, it is important to highlight the ones located in the ranges: (i) $1686-1677 \mathrm{~cm}^{-1}$, (ii) $1634-1619 \mathrm{~cm}^{-1}$, and (iii) $3227-3080 \mathrm{~cm}^{-}$ 1. The first absorption signal is attributed to the stretching $(v) \mathrm{C}=\mathrm{O}$ of a free carbonyl group, the second one to the stretching $(v) \mathrm{C}=\mathrm{O}$ of another carbonyl group bonded intramolecularly with a hydrogen atom from an amino group, and the third one to the stretching $(v) \mathrm{N}-\mathrm{H}$ of the mentioned amino group.
Table 1. Identification of absorption peaks in the FTIR spectra of compounds 4-NANM-E and 4-methyl-NANM.

\begin{tabular}{|c|c|}
\hline Peak (cm-1) & Attributed to \\
\hline $3227-3080$ & Stretching $(v) \mathrm{N}-\mathrm{H}$ of amino group \\
\hline $3000-2850$ & Stretching $(v) \mathrm{CH}_{2}$ and $\mathrm{CH}_{3}$, symmetric and unsymmetric \\
\hline $1686-1677$ & Stretching $(v) \mathrm{C}=\mathrm{O}$ of free carbonyl \\
\hline $1634-1619$ & Stretching $(v) \mathrm{C}=\mathrm{O}$ of chelated carbonyl (with $\mathrm{NH}$ group) \\
\hline $1582-1578$ & Bending $(\delta) \mathrm{N}-\mathrm{H}$ and stretching $(v) \mathrm{C}=\mathrm{C}$ (ring, vinyl) \\
\hline 1515 & Stretching $(v) \mathrm{C}-\mathrm{NO}_{2}$ unsymmetric \\
\hline $1339-1335$ & Stretching $(v) \mathrm{C}-\mathrm{NO}_{2}$ symmetric \\
\hline $1246-1233$ & Stretching $(v) \mathrm{C}-\mathrm{NH}$ and $(v)$ CO-O unsymmetric \\
\hline 1104 & Stretching $(v)$ COO-C unsymmetric \\
\hline
\end{tabular}

In the ${ }^{1} \mathrm{H}-\mathrm{NMR}$ spectrum of Figure 2, the signal generated by the amino group was observed between 10.74-10.78 ppm (low field) as a doublet ( $\mathrm{J}_{\mathrm{NH}}$ ${ }_{\mathrm{CH}}: 13.4 \mathrm{~Hz}$ ) due to the coupling between the proton $\mathrm{NH}$ and the vinyl proton at the adjacent alpha carbon. The doublet observed between 8.39-8.42 ppm $\left(\mathrm{J}_{\mathrm{NH}=\mathrm{CH}}: 13.4 \mathrm{~Hz}\right)$ is attributed to the vinyl proton. The high value of coupling constant $\left(\mathrm{J}_{\mathrm{NH},=\mathrm{CH}}: 13.4 \mathrm{~Hz}\right)$ suggests a "trans" relationship between the vinyl and amino protons, and the hindrance to rotation around the $\mathrm{C}-\mathrm{N}$ bond due to the hydrogen bond between - $\mathrm{NH}$ and one of the carbonyls $(\mathrm{C}=\mathrm{O}) \cdot{ }^{19}$ Both the free and chelated carbonyl groups were detected in the ${ }^{13} \mathrm{C}$ NMR spectrum as signals at 164.7 and $166.5 \mathrm{ppm}$, respectively (Figure 3 ). The other signals appearing in IR (Figure 1, Table 1), ${ }^{1} \mathrm{H}-\mathrm{NMR}$ (Figure 2) and ${ }^{13} \mathrm{C}$-NMR (Figure 3 ) spectra of the synthesized products confirmed the presence of the functional groups characteristic of alkyl 4-NANM and its molecular formula.

The formation of 4-NANM-methyl suggested that the ethyl ester groups of EMA underwent nucleophilic substitution of ethoxy groups by methoxy radicals (methanolysis) during the reaction with 4-nitroaniline in methanol$\mathrm{KOH}$. This was confirmed after analyzing by proton NMR the reaction product formed between EMA and methanol-KOH in the absence of 4-nitroaniline. The product of this reaction (filtered solid, washed with methanol, and vacuum dried) showed only two proton signals (at $\left.\mathrm{D}_{2} \mathrm{O}\right)$ which corresponds to the vinyl proton $(9.17 \mathrm{ppm})$ and the six methyl protons $(3.64 \mathrm{ppm})$, and this not only indicates the occurrence of methanolysis of EMA, but also the formation of the dimethyl 2-(hydroxymethylene)malonate potassium salt (Figure 4), which was soluble only in water and DMSO. 


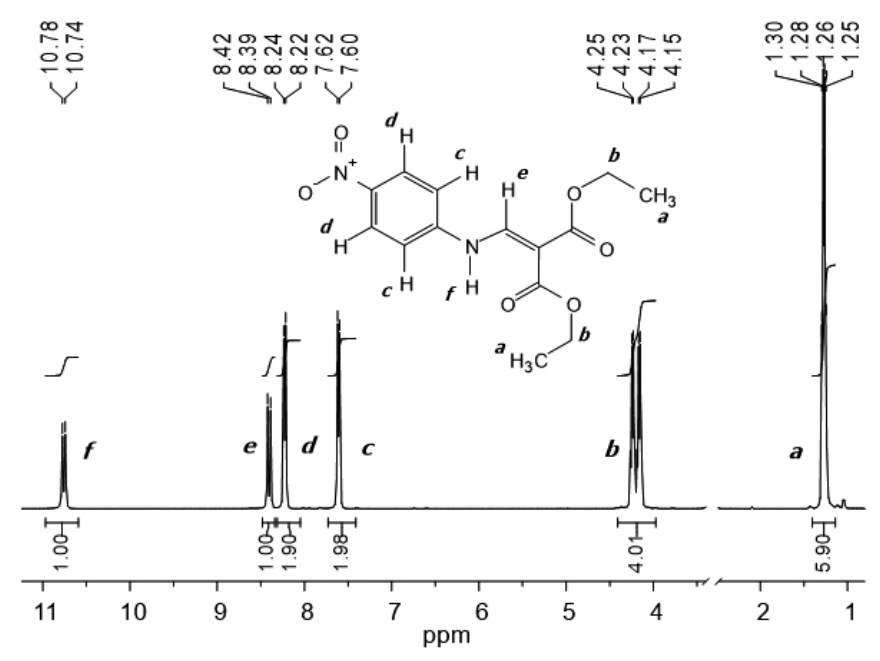

Figure 2. ${ }^{1} \mathrm{H}-\mathrm{NMR}$ spectrum of 4-NANM-E obtained in the 2-propanol$\mathrm{KOH}$ system. The solvent (DMSO- $d_{6}$ ) signal was removed for clarity.

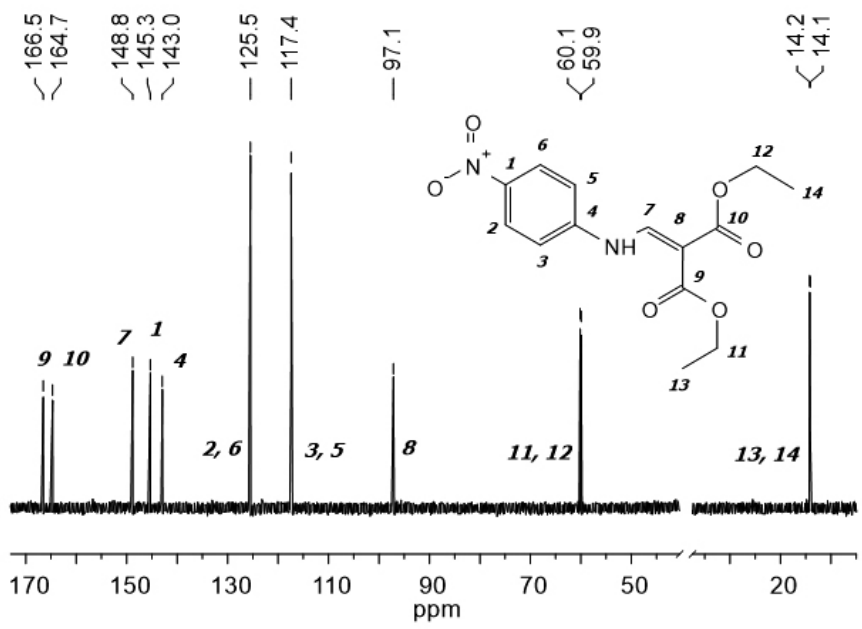

Figure 3. ${ }^{13} \mathrm{C}$-NMR spectrum of 4-NANM-E obtained in the 2-propanol$\mathrm{KOH}$ system. The solvent (DMSO- $d_{6}$ ) signal was removed for clarity.

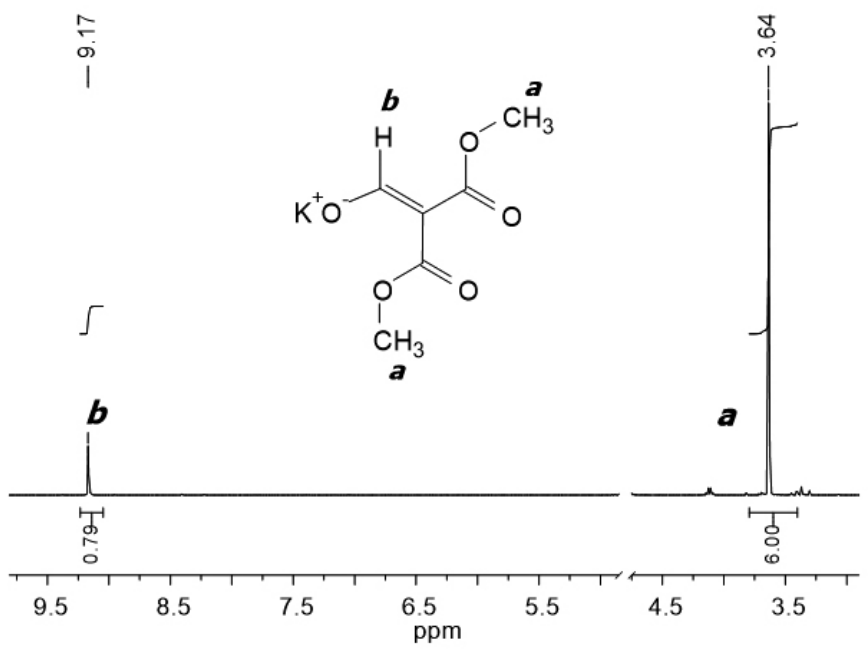

Figure 4. ${ }^{1} \mathrm{H}-\mathrm{NMR}$ spectrum in $\mathrm{D}_{2} \mathrm{O}$ of the potassium salt of dimethyl 2-(hydroxymethylene) malonate. The solvent signal was removed for clarity.
Although the present study does not attempt to explain the reaction mechanism by which alkyl 4-NANM is obtained under the conditions of alcohol$\mathrm{KOH}$ as solvent and without heating, it is likely that the 2-(hydroxymethylene) malonate potassium salt is initially formed and then it reacts with 4-nitroaniline following nucleophilic vinyl substitution. ${ }^{12}$ This argument coincides with the Mulveyl's method for the preparation of anilinomethylenmalonates, which use an alkyl 2-(hydroxymethylene)malonate salt and a substituted aniline as initial reagents, but in this case, the reaction solvent consists of a strong acid dissolved in short chain aliphatic alcohol and the reaction is much slower. ${ }^{20}$ The synthesis of diethyl/dimethyl 2-(hydroxymethylene)malonate by subjecting the respective alkyl alkoxymethylenemalonates to mild basic conditions with aqueous $\mathrm{NaOH},{ }^{21} \mathrm{KOH}$-water/diethyl ether, ${ }^{22}$ or $\mathrm{KOH}$-methanol/diethyl ether ${ }^{2 .}$ were already reported but these studies are intended to obtain the enol derivative of the alkoxymethylenemalonate instead of anilinomethylenmalonates.

A possible explanation regarding the occurrence of methanolysis or transesterification of EMA is the lower basicity of methanol compared to that of $\mathrm{KOH}$ (pKa: 15.3 vs 15.7) which implies a greater capacity of the latter to deprotonate methanol molecules, generating enough methoxide anions which are the nucleophiles that attack the carbonylic carbons of EMA. In addition, it has been confirmed from the previous studies that the nucleophilic character of the alkoxide anions decreases as the length of the alkyl chain increases, and thus increase the steric hindrance for a nucleophilic acyl substitution to take place. ${ }^{24}$ The transesterification of EMA did not occur in the reactions carried out with ethanol, 2-propanol, 2-butanol and 2-pentanol due to the low nucleophilicity and more basic nature than that of $\mathrm{KOH}$ (pKa: 16-18 vs 15.7) which makes more difficult to deprotonate by the hydroxide compared to methanol, avoiding the production of alkoxide radicals.

The yields of the 4-NANM-E synthesis in the different alcohols were the following: $45 \%$ in ethanol, $48 \%$ in 2-propanol, $53 \%$ in 2-butanol and $47 \%$ in 2-pentanol. Only $16 \%$ yield of 4-NANM-methyl was obtained for the reaction performed in methanol. The advantages of this 4-NANM-E synthesis method are simply ease, less reaction time and highly pure product, which is necessary if it is desired to obtain good yields of the respective quinolone by the GouldJacob reaction. ${ }^{15}$

\section{CONCLUSIONS}

An efficient and a fast method for the synthesis of 4-NANM-E at room temperature has been developed using equimolar amounts of EMA and 4-nitroaniline. The best yields are obtained using the alcohols: 2-butanol (53\%), 2-propanol (48\%), 2-pentanol (47\%), and ethanol (45\%). The easy purification, less time, and less cost of this method makes it an excellent alternative to produce 4-NANM-E on an industrial scale.

\section{ACKNOWLEDGMENTS}

Hernan Valle is grateful to FONDECYT for the postdoctoral Grant $\mathrm{N}^{\circ}$ 3160296 .

\section{REFERENCES}

1.- M. Artico, A. Mai, G. Sbardella, S. Massa, C. Musiu, S. Lostia, F. Demontis, P. La Colla, Bioorganic Med. Chem. Lett. 9, 1651, (1999).

2.- M.V. Shul'gina, N.I. Fadeeva, T.N. Bol'shakova, I.B. Levshin, R.G Glushkov, Pharm. Chem. J. 33, 343, (1999).

3.- S. Sarkar, P. Ghosh, A. Misra, S. Das, Synth. Commun. 45, 2386, (2015).

4.- T. Stärhfeldt, Patent US006172232B1, 2001

5.- J.A. Tucker, V.A. Vaillancourt, J.W. Strohbach, K.R. Romines, M.E. Schnute, M.M. Cudahy, S. Thaisrivongs, S.R. Turner, Patent US006093732A, 2000.

6.- J.-F. He, L.-H. Yun, R.-F. Yang, Z.-Y. Xiao, J.-P. Cheng, W.-X. Zhou, Y.-X. Zhang, Bioorg. Med. Chem. Lett. 15, 2980, (2005).

7.- B. Lucero, C. Gomes, I. Frugulhetti, L. Faro, L. Alvarenga, M. De Souza, T. De Souza, V. Ferreira, Bioorganic Med. Chem. Lett.16, 1010, (2006).

8.- M.P. Moyer, F.H. Weber, J.L. Gross, J.W. Isaac, R. Fort, Bioorg. Med Chem. Lett. 2, 1589, (1992).

9.- F. Boechat, C. Sacramento, A. Cunha, F. Sagrillo, C. Nogueira, N. Fintelman-Rodrigues, O. Santos-Filho, C. Riscado, L. Forezi, L. Faro, L. Brozeguini, I. Marques, V. Ferreira, T. Souza, M. De Souza, Bioorganic Med. Chem. 23, 7777, (2015).

10.- A.P. Kaplan, V. Gupta, J.W.F. Wasley, Patent US 20080306049A1, 2008. 
11.- D. Yang, L. Arifhodzic, C.R. Ganellin, D.H. Jenkinson, Eur. J. Med. Chem. 63, 907, (2013)

12.- C. Oh, I. Yi, K.P. Park, J. Heterocycl. Chem. 31, 841, (1994).

13.- H. Agui, T. Mitani, M. Nakashita, T. Nakagome, J. Heterocyclic Chem. 8, 357, (1971)

14.- B. Riegel, G.R. Lappin, B.H. Adelson, R.I. Jackson, C.J. Albisetti, R.M. Dodson, R.H. Baker, J. Am. Chem. Soc. 68, 1264, (1946).

15.- G.F. Duffin,; J.D. Kendall, J. Chem. Soc. 893, (1948).

16.- D. Tarabová, V. Milata, J. Hanusek, Acta Chim. Slovaca. 6, 73, (2013).

17.- K.-W. Kim, H.-J. Lee, J.-I. Jo, T.-W. Kwon, Bull. Korean Chem. Soc. 31, $1155,(2010)$.

18.- C. Leonelli and P. Veronesi, In Production of Biofuels and Chemicals with
Microwave, Z. Fang, R.L. Smith Jr., X. Qi, Eds. Springer Netherlands, Dordrecht, 2015; pp. 17-40.

19.- A. Gómez-Sanchez, E. Sempere, J. Bellanato, J. Chem. Soc. Perkin Trans 2. 3, 561, (1981)

20.- D. M. Mulvey, R.J. Tull, L.M. Weinstock, Patent US3515745A, 1970.

21.- N. Katagiri, H. Akatsuka, T. Haneda, C. Kaneko, A. Sera, J. Org. Chem. 53, 5464, (1988)

22.- S. Antus, F. Boross, M. Nógrádi, Justus Liebigs Ann. Chem. 1, 107, (1978).

23.- I.A. Wolff, D.W. Olds, G.E. Hilbert, Synthesis (Stuttg). 9, 732, (1984).

24.- D. Kusdiana, S. Saka, Fuel. 80, 693, (2001). 